BIO Web of Conferences 6,01003 (2016)

DOI: $10.1051 /$ bioconf/20160601003

C) Owned by the authors, published by EDP Sciences, 2016

\title{
A direct and at nanometer scale study of electrical charge distribution on membranes of alive cells
}

\author{
Christian Marlière ${ }^{a}$ \\ Institut des Sciences Moléculaires d'Orsay (ISMO), Université Paris-Sud, Université Paris-Saclay, 91405 Orsay \\ Cedex, France
}

\begin{abstract}
In this paper is presented an innovative method to map in-vivo and at nanometer scale the electrical charge distribution on membranes of alive cells. It relies on a new atomic force microscopy (AFM) mode based on an electro-mechanical coupling effect. Furthermore, an additional electrical signal detected by both the deflection of the AFM cantilever and simultaneous direct current measurements was detected at low scanning rates. It was attributed to the detection of the current stemming from ionic channels. It opens a new way to directly investigate in situ biological electrical surface processes involved in bacterial adhesion, biofilm formation, microbial fuel cells, etc.
\end{abstract}

${ }^{\text {a }}$ Corresponding author: christian.marliere@u-psud.fr 